\title{
The RIFLE criteria: Are the foundations robust?
}

Pierre Delanaye, $\mathrm{MD}^{1}$; Jean-Marie Krzesinski, $\mathrm{MD}, \mathrm{PhD}^{1}$; Etienne Cavalier, $\mathrm{MD}^{2}$; Bernard Lambermont, MD, $\mathrm{PhD}^{3}$

${ }^{I}$ Department of Nephrology-Dialysis, University of Liège, University Hospital of Sart Tilman, Liège, Belgium

${ }^{2}$ Department of Clinical Chemistry, University of Liège, University Hospital of Sart Tilman, Liège, Belgium

${ }^{3}$ Medical Intensive Care Unit, Department of Medicine, University of Liège, University Hospital of Sart Tilman, Liège, Belgium

\section{To the Editor:}

We read with interest the article by Drs. Ostermann and Chang (1) about the RIFLE criteria as a classification of renal failure and as a prognostic factor in intensive care. We would like to make some comments, also valid for the other articles published on this topic. One important criterion in the RIFLE classification is the glomerular filtration rate (GFR). It is even the sole criterion used in this study because urine data were not available. In all studies published on the RIFLE criteria, GFR is not measured but estimated by the "modification of diet in renal disease" (MDRD) equation. This equation is based on four variables: gender, age, ethnicity, and serum creatinine. Among these variables, only serum creatinine can change abruptly. Therefore, using MDRD (or any other creatinine-based formula) in the intensive care setting is very questionable. The MDRD equation has been poorly validated in intensive care patients. Hoste et al. (2) showed that the MDRD equation was not adequate to estimate GFR, even if this study included few patients and used a questionable reference method for the GFR measurement (1-hr creatinine clearance). Once again, serum creatinine concentration, the most important variable of the MDRD equation, is also dependent on muscular mass, and patients hospitalized in intensive care units are prone to present decreased muscular mass. Consequently, serum creatinine may remain low or not increase as much as expected even if renal failure occurs. As a consequence, the MDRD equation may potentially overestimate true GFR. Such an overestimation has been clearly demonstrated in other patients with decreased muscular mass like heart transplant patients or patients with low body weight $(3,4)$. In the same way, even an increase in creatinine concentration of only $0.3 \mathrm{mg} / \mathrm{dL}$ could correspond to a large decrease in GFR in patients with loss of muscular mass and explain why such a limited modification of serum creatinine is linked to mortality (1). The inability of creatinine and MDRD to correctly estimate GFR in patients with decreasing muscular mass may also partially explain differences observed in the incidence of renal failure between studies with different populations and between studies using the GFR or the urine criteria.

It is important to remind intensivists that the MDRD equation might strongly overestimate GFR in intensive care unit patients. Moreover, this equation lacks precision when applied to populations with normal renal function and creatinine values (5). We agree that the RIFLE classification has great advantages, especially in term of standard definition and classification system. Nevertheless, there are limitations to the use of the MDRD equation in the GFR criteria. Using MDRD can cause misclassification of several patients. More studies using a reference method for GFR measurement (such as iohexol urine clearance) or new plasma or urine renal biomarker (such as cystatin C) seem necessary.

\section{References}

1. Ostermann M, Chang RW: Acute kidney injury in the intensive care unit according to RIFLE. Crit Care Med 2007; 35:1837-1843

2. Hoste EA, Damen J, Vanholder RC, et al: Assessment of renal function in recently admitted critically ill patients with normal serum creatinine. Nephrol Dial Transplant 2005; 20: 747-753

3. Delanaye P, Nellessen E, Grosch S, et al: Creatinine-based formulae for the estimation of glomerular filtration rate in heart transplant recipients. Clin Transplant 2006; 20:596-603

4. Froissart M, Rossert J, Jacquot C, et al: Predictive performance of the modification of diet in renal disease and Cockcroft-Gault equations for estimating renal function. J Am Soc Nephrol 2005; 16:763-773

5. Delanaye P, Cavalier E, Krzesinski JM, et al: Why the MDRD equation should not be used in patients with normal renal function (and normal creatinine values). Clin Nephrol 2006; 66:147-148 
